\title{
An initial meteoroid stream survey in the southern hemisphere using the Southern Argentina Agile Meteor Radar (SAAMER)
}

\author{
D. Janches $^{\mathrm{a}, *}$, J.L. Hormaechea ${ }^{\mathrm{b}}$, C. Brunini ${ }^{\mathrm{c}}$, W. Hocking ${ }^{\mathrm{d}}$, D.C. Fritts ${ }^{\mathrm{e}}$ \\ ${ }^{a}$ Space Weather Lab., Mail Code 674, GSFC/NASA, Greenbelt, MD 20771, United States \\ ${ }^{\mathrm{b}}$ Estacion Astronomica Rio Grande, Rio Grande, Tierra del Fuego, Argentina \\ ${ }^{\mathrm{c}}$ Departamento de Ciencias Astronomicas y Geofisicas, Universidad Nacional de La Plata, La Plata, Argentina \\ ${ }^{\mathrm{d}}$ Department of Physics and Astronomy, University of Western Ontario, Canada \\ ${ }^{\mathrm{e}}$ Gats Inc., Boulder, CO 80302, United States
}

\section{A R T I C L E I N F O}

\section{Article history:}

Received 20 August 2012

Revised 18 December 2012

Accepted 24 December 2012

Available online 16 January 2013

\begin{abstract}
A B S T R A C T
We present in this manuscript a 4 year survey of meteor shower radiants utilizing the Southern Argentina Agile Meteor Radar (SAAMER). SAAMER, which operates at the southern most region of South America, is a new generation SKiYMET system designed with significant differences from typical meteor radars including high transmitted power and an 8-antenna transmitting array enabling large detected rates at low zenith angles. We applied the statistical methodology developed by Jones and Jones (Jones, J., Jones, W. [2006]. Month. Not. R. Astron. Soc. 367, 1050-1056) to the data collected each day and compiled the results into 1 composite representative year at $1^{\circ}$ resolution in Solar Longitude. We then search for enhancements in the activity which last for at least 3 days and evolve temporally as is expected from a meteor shower. Using this methodology, we have identified in our data 32 shower radiants, two of which were not part of the IAU commission 22 meteor shower working list. Recently, SAAMER's capabilities were enhanced by adding two remote stations to receive meteor forward scatter signals from meteor trails and thus enable the determination of meteoroid orbital parameters. SAAMER started recording orbits in January 2012 and future surveys will focus on the search for unknown meteor streams, in particular in the southern ecliptic sky.
\end{abstract}

Published by Elsevier Inc.

\section{Introduction}

The collision of asteroids and disintegration of comets is the main source of dust in the Solar System. These processes give rise to a thick circumsolar disk of small debris known as the Zodiacal Dust Cloud (ZDC). Several physical effects produced by larger Solar System bodies result in the dust having relatively short lifetimes maintaining somewhat a balance in their distribution preventing this cloud from becoming dustier. For example, they can be ejected from the Solar System by Jupiter, thermally obliterated by the Sun, or physically fragmented by additional collisions amongst them. Also, a portion of the cloud is swept by the planets, and for the case of those with atmospheres will produce the more familiar phenomena of ionization and light production that is termed meteor. We now know that similar processes occur in other systems as circumstellar disks of dust have been observed, for example, around Beta Pitcoris and Formalhaut. Thus understanding the nature of ZDC can shed light to the history and development of the Solar Sys-

\footnotetext{
* Corresponding author.

E-mail addresses: diego.janches@nasa.gov (D. Janches), jlhor@earg.gov.ar (J.L Hormaechea), claudiobrunini@yahoo.com (C. Brunini), whocking@uwo.ca (W. Hocking), dave@gats-inc.com (D.C. Fritts).
}

tem as well as extra solar planetary environments (Malhotra, 1995; Johansen et al., 2007; Walsh et al., 2011; Nesvorný et al., 2010; Wiegert et al., 2009).

Meteor showers, in particular, are an excellent tool for these studies because they are the direct result of cometary mass loss through their approach to the inner Solar System. Sporadic meteors, on the other hand, are characterized by orbits which have evolved significantly from their original at the moment of ejection from their parent body and thus have lost their history. This implies that, as oppose to showers, they cannot be associated with a particular parent body and/or other particles from the same parent. Thus meteor showers are an excellent tool to constraint dynamical models of cometary evolution, both orbital and physical. Surveying and studying meteor showers can shed light to cometary composition and structure, dust ejection speeds, parent body masses and some times even constrain their period in the Solar System (Sykes and Walker, 1992; Jenniskens, 2008; Wiegert and Brown, 2004; Borovička et al., 2005). Brown et al. (2008) provides a detail account of known previous shower surveys, and as discussed by the authors, meteor radars are an effective tool to perform these surveys, in particular for smaller particles. It is also shown in that work that most of the known surveys were performed in the northern hemisphere with only 10\% taking place 
at southern latitudes, the latest of which involves observations performed during a 4 year period prior to 2000 in which only six streams were found (Galligan and Baggaley, 2002a,b). A more recent survey utilizing 2 years of observations was reported by Younger et al. (2009) in which over 30 showers were identified. The continual survey of meteor showers is important because it provides information on the sometimes cyclic annual strength in the activity of showers, and thus constrain models of dust evolution in the Solar System. A good example is the Leonids meteor shower which have produced a number of noteworthy storms through out history, particularly those in 1833, 1868 and most recently in 1997-1998 period (Jenniskens, 2006) but currently its activity is very weak.

For the study presented here we focus on the utilization of the Southern Argentina Agile Meteor Radar (SAAMER) and the application of the statistical method developed by Jones and Jones (2006) to estimate meteor shower radiants in single-station data collection mode. We report a 4 year survey of meteor shower radiants utilizing a new generation SKiYMET system designed with significant differences from typical meteor radars including high transmitted power and an 8-antenna transmitting array enabling large detected rates at low zenith angles. A full description of the new meteor radar system is provided in Section 2 while a description of our searching technique is provided in Section 3. The results are discussed in Section 4.

\section{SAAMER: system description}

The Southern Argentina Agile Meteor Radar (SAAMER) is a SKiYMET system deployed at the Estacion Astronomica Rio Grande (EARG) in the city of Rio Grande $\left(53.8^{\circ} 45^{\prime} 8^{\prime \prime} S\right.$; $\left.67^{\circ} 45^{\prime} 5^{\prime \prime} \mathrm{W}\right)$, province of Tierra del Fuego, Argentina. The system, which has being operational continuously since May, 2008 was enhanced, relative to standard meteor radars, in order to enable Gravity Wave (GW) momentum flux measurements in the Mesosphere and Lower
Thermosphere (MLT) atmospheric region (Fritts et al., 2010b,a). These enhancements were driven by two radical changes from the typical systems: (1) significantly higher meteor counts (i.e. by at least an order of magnitude) and (2) a need for the majority of meteor detections to be at small zenith (high elevation) angles. Both needs were addressed with SAAMER, which was designed for greatly enhanced transmitter peak power $(60 \mathrm{~kW}$, rather than $6-$ $20 \mathrm{~kW}$ used by most meteor radar systems) and uses a transmitter antenna (specially designed by Mardoc Inc.) composed of eight (instead of one) 3-element crossed yagis (rather than 2-element) arranged in a circle of diameter $27.6 \mathrm{~m}$ (Fig. 1). SAAMER's operating frequency and bandwidth are 32.55 and $0.3 \mathrm{MHz}$, respectively. In the normal mode of operation, as it is the case for the data presented in this work, SAAMER transmits a 2-km long pulse with opposite phasing of every other yagi, directing the majority of radar power into eight beams at $45^{\circ}$ azimuth increments with peak power at $\sim 35^{\circ}$ off zenith. This results in a majority of meteor detections at off-zenith angles between $15^{\circ}$ and $50^{\circ}$. The receiving array is formed by the typical five-antenna interferometer arrangement (Hocking et al., 1997; Jones et al., 1998), all of which are also 3-element crossed yagis enabling redundant meteor position definition with errors less than $0.5^{\circ}$. Similarly as for the Canadian Meteor Orbits Radar (CMOR; Brown et al., 2008), SAAMER uses the basic echo detection and analysis algorithms for the SKiYMET systems developed by Hocking et al. (2001). Together, these upgrades increase the power at the small zenith angles of interest by $\sim 23 \mathrm{~dB}$ and the near-zenith (i.e. elevation angles greater than $50^{\circ}$ ) meteor counts by $\sim 20$ integrated over all azimuths. This is shown in Fig. 2 where daily specular underdense meteor trail detected rates are shown for the nearly 4 year long period of observations utilized in this study. During the first 16 months of operation, SAAMER transmitted a monopulse at a pulse repetition frequency (PRF) of $2140 \mathrm{~Hz}$, resulting in an excess of 10,000 specular trail detections daily (top panel in Fig. 2). In September of 2009, however, we changed the transmitting scheme to a 2-bit
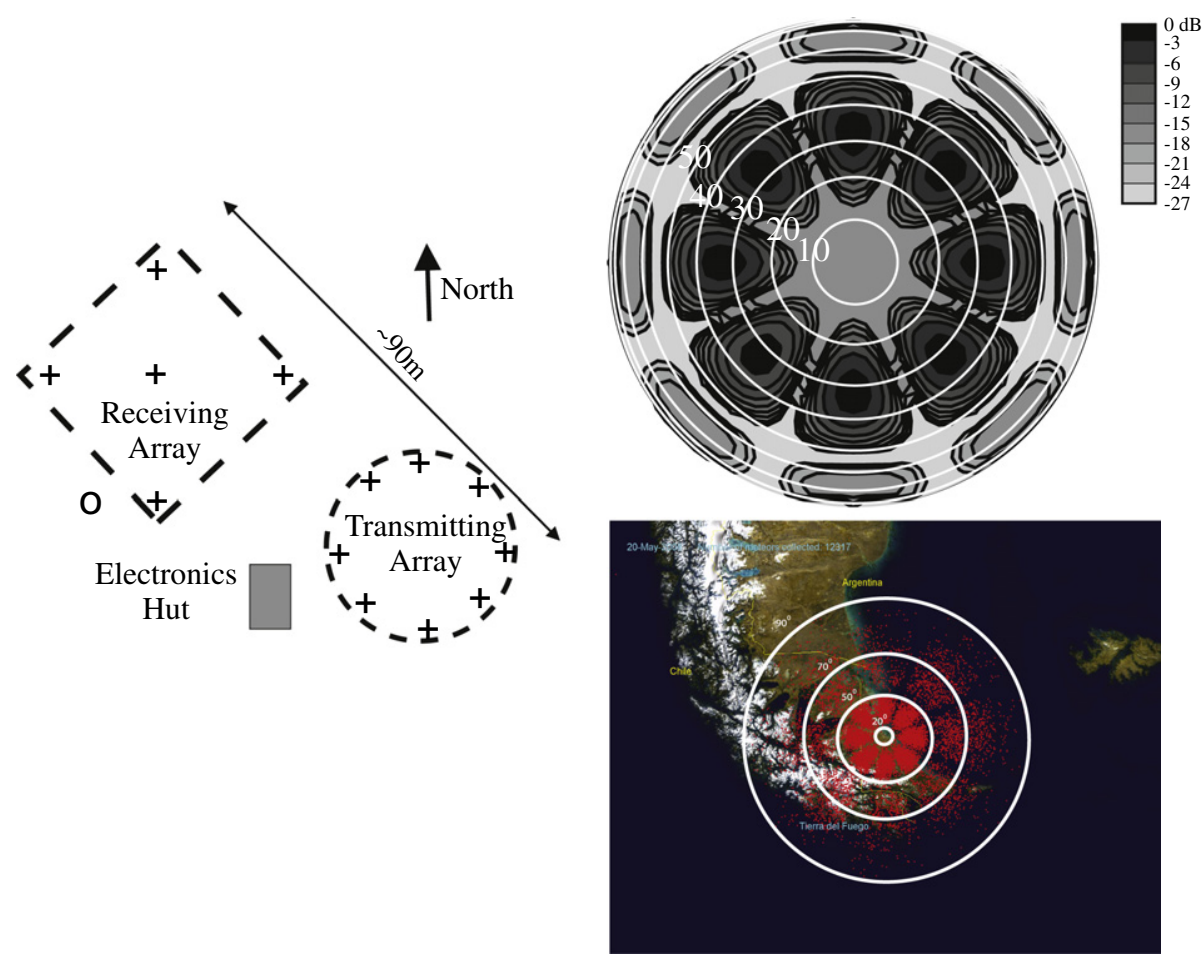

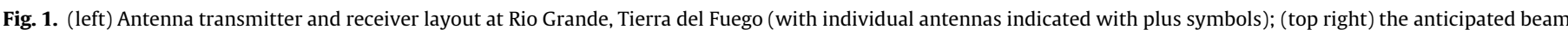

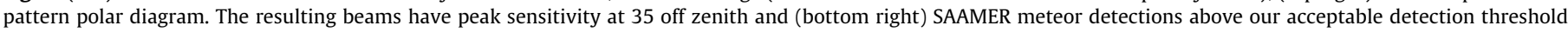
(total = 12,317) for 20 May 2008 . 

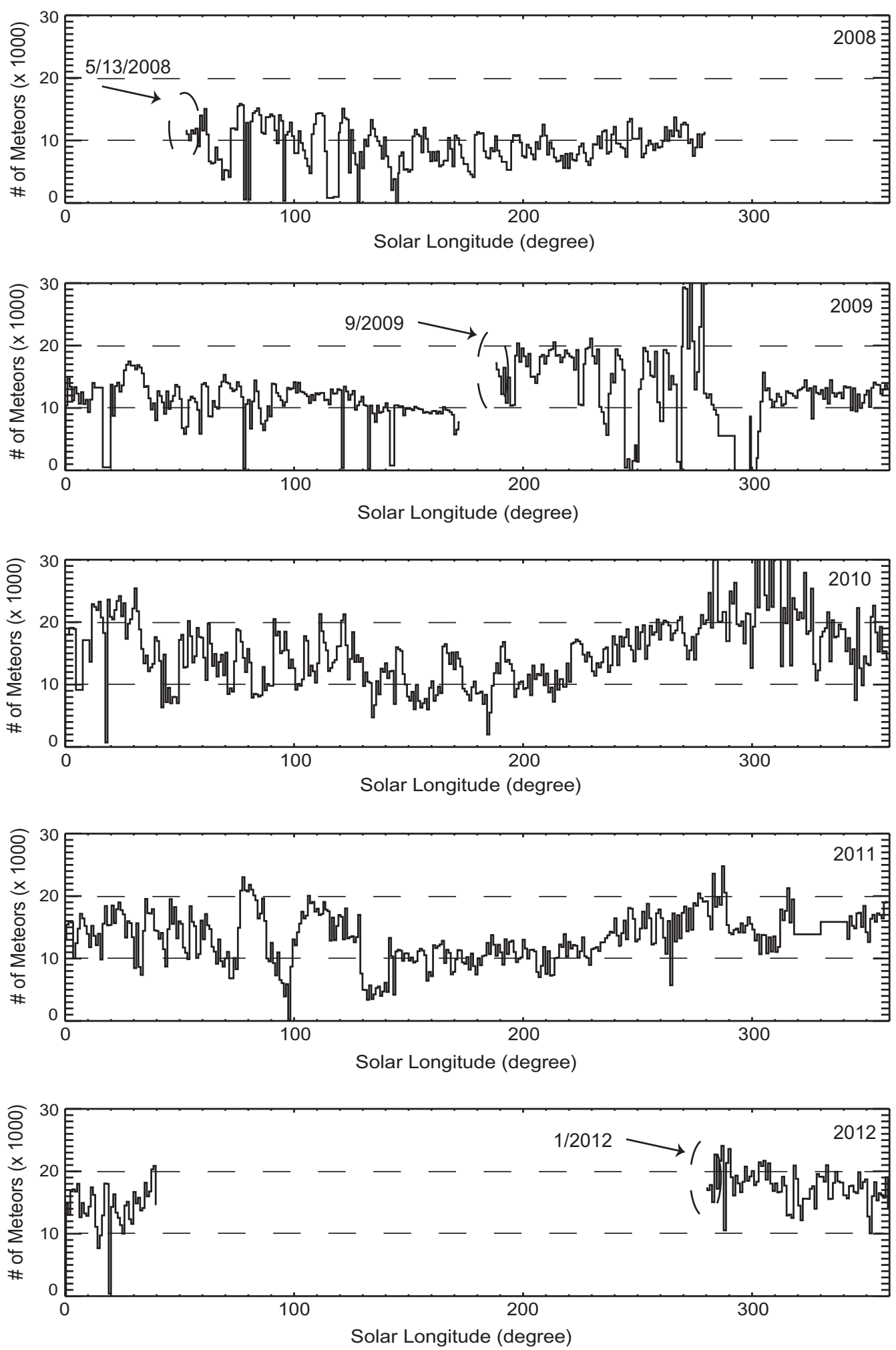

Fig. 2. Meteor rates detected by SAAMER in the normal mode of operation for the $\sim 4$ years of data utilized in this study.

coded pulse at a PRF of $1765 \mathrm{~Hz}$ resulting in a $40 \%$ increase in the daily counts. Overall, the detected rates are $\sim 2-3$ times larger than for example rates detected with CMOR (Brown et al., 2008). This is particularly remarkable considering that, most of the detections occur overhead, opposite to most of meteor radar designs. A comparison between SAAMER's meteor detection performance and that of typical systems is presented in Fritts et al. (2012). In August 2010 , we enhanced the capabilities of the radar by adding two remote stations to receive meteor forward scatter signals from meteor trails. The information provided by the outlying receiving stations enables the determination of meteoroid trajectories and speeds of meteoroids and thus leads to the determination of their 
orbital parameters (Baggaley et al., 1994; Brown et al., 2008). Orbital information have been recorded since January 2012 and will be reported in future works.

\section{Data analysis}

Traditional VHF meteor radars (often called all-sky radars) primarily detect the specular reflection of meteor trails traveling perpendicular to the line of sight of the scattering trail. This implies that their velocity vector (traveling direction) must fall within the plane perpendicular to the line between the radar and the point in the sky where the echo was recorded (line-of-sight). As pointed out earlier, this information is accurately recorded using the interferometer receiving array. In other words, all possible meteor radiants will fall within a circle perpendicular to the meteor line-of-sight. If there are meteors belonging to a particular stream, the circles defined by these particular meteors will all intersect in one common point producing an enhancement above the noise. By locating these enhancements projected in equatorial coordinates (i.e. Right Ascention RA and Declination $\delta$ ), meteor shower radiants can be determined. For this purpose Jones and Morton (1977) developed a statistical technique, later improved by Jones and Jones (2006), which looks at each possible radiant and count the detections in a band perpendicular to each of them. This is performed by defining acceptance bands which are convolved with the filter function given by

$\omega(\theta)= \begin{cases}1-6\left(\frac{\theta}{\delta \theta}\right)^{2}+5\left(\frac{\theta}{\delta \theta}\right)^{4} & \text { for }|\theta| \leqslant \delta \theta \\ 0 & \text { for }|\theta|>\delta \theta\end{cases}$

where $\theta$ is the angular separation to the center of the acceptance band. As suggested by Jones and Jones (2006) we implement this filter using $\delta \theta=4^{\circ}$ and searching all possible directions which lay above the local horizon with a $\sim 1.8^{\circ}$ resolution in both equatorial coordinates.

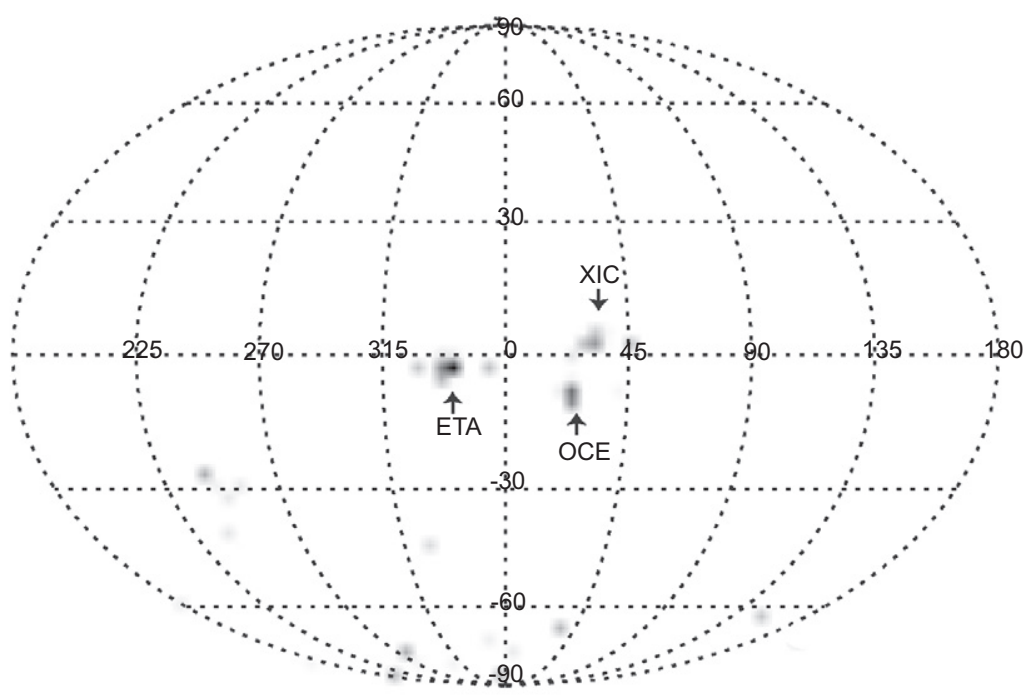

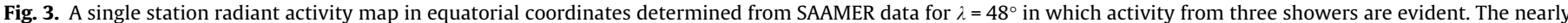
4 year of data are included in this map.

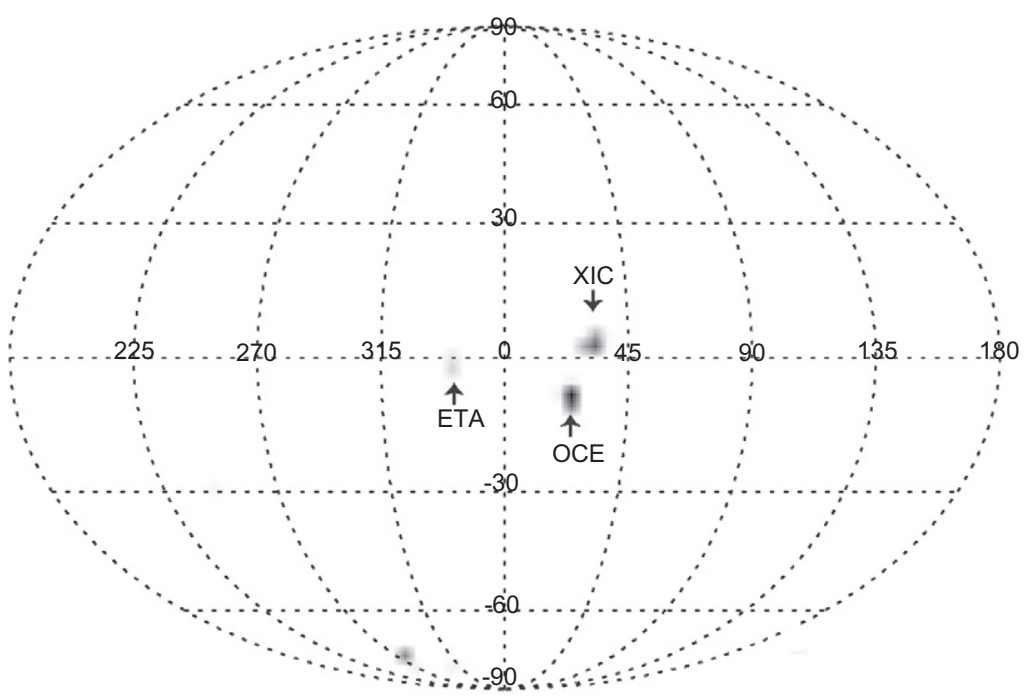

Fig. 4. 2D Gaussian fitted results to the data shown in Fig. 3 
For this work and similarly to Brown et al. (2008) we focus on defining showers that are active year-to-year and thus we combined all data from all years into a single equivalent solar year, by binning the echo data by Solar Longitude and constructing radiant maps in equatorial coordinates. Fig. 3 shows an example resulting from this methodology where enhancements due to the presence of three different showers at Solar Longitude $(\lambda)$ equal to $48^{\circ}$ are evident. These are the $\eta$ Aquariids (ETA), Daytime $\xi$ Cetids (XIC) and Southern Daytime $\omega$ Cetids (OCE). Note that due to the high southern latitude location, SAAMER is prevented to see declinations much higher than $\sim 35^{\circ}$, conjugate to CMOR which efficiently detects meteors which radiant declination lies polarward $\sim-35^{\circ}$.

As a first step in the search and identification of radiant enhancements, we use the ETA and Southern June Aquiliids (SZC), two of the strongest annual showers, in these radiant maps to define the minimum relative strength with respect to the background noise that an enhancement needs to have in order to be consider a potential candidate. This threshold is determined by the relative strength of these showers during the first and last days during which they are visible with respect to the sporadic background. Each map for each degree of Solar Longitude was examined by fitting a 2D Gaussian curve of $\sim 4^{\circ} \times 4^{\circ}$ on all enhancements where the maxima was equal or greater than this threshold. Fig. 4 shows the fitted results for the data corresponding to Fig. 3 where it can be observed that not only the three showers were successfully identified, but also most of the noise is removed. A list of potential radiants was compiled for each Solar Longitude. Following this step, we search in the resulting list of potential radiants and select those which were present for a minimum of $3^{\circ}$ in Solar Longitude and within $2^{\circ}$ in RA and $\delta$ between continuous bins. We then recorded all these points together as a possible
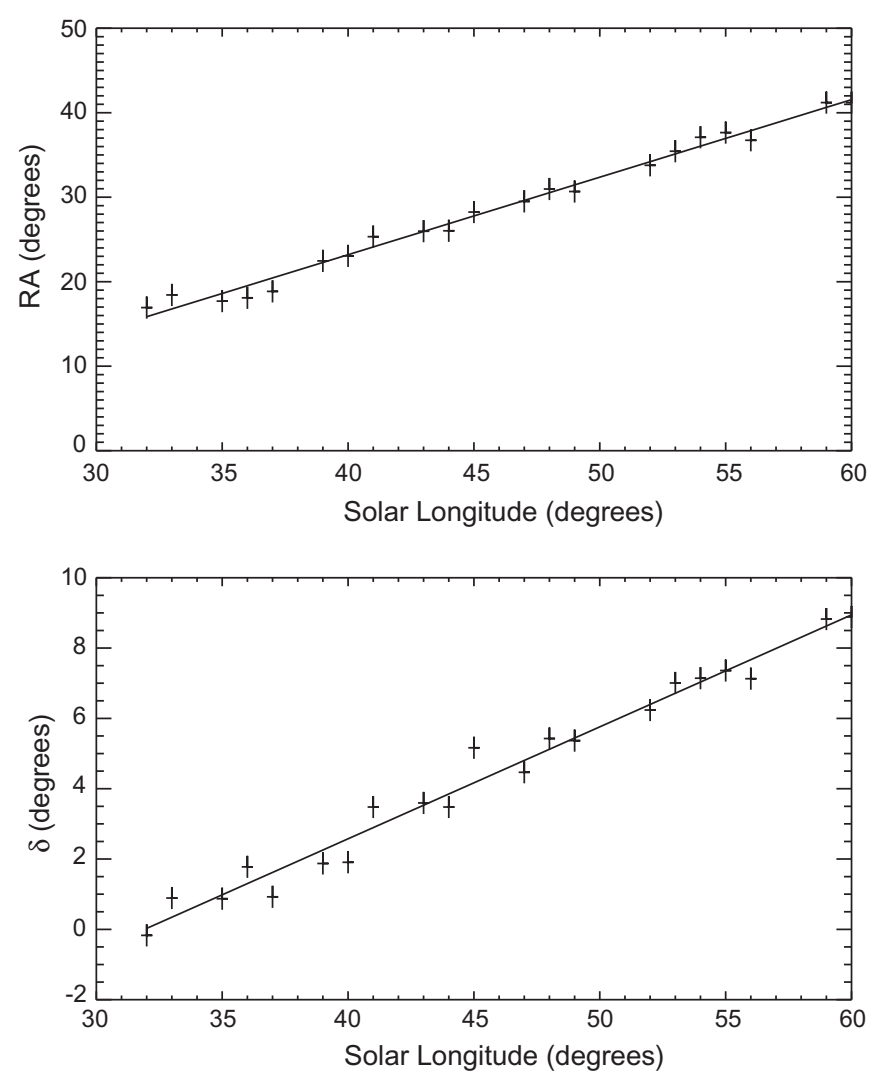

Fig. 5. Activity plots for the OCE meteor shower. linked radiant constituting a shower. The final step on the search is to visually examine these linked radiants and those showing consistent positive drifts in RA, and consistent drifts throughout their activity period in $\delta$ were singled out for identification. Fig. 5 shows the time evolutions of RA and $\delta$ for OCE as well as a line fit used to calculate the drift.

\section{Results and discussions}

Utilizing the methodology described in Section 3, we identified over 60 radiant candidates. In order to unequivocally identify a meteor stream, however, it is crucial to determine geocentric velocity and orbital information which are not accessible for the data presented in this survey. Due to this limitation, we only focus on reporting those radiants for which identification was possible, which was carried out initially by comparing our results with the Meteor Working List (MWL) reported by the International Astronomical Union (IAU) Meteor Data Center (Jopek and Jenniskens, 2011). For this, we search for values in $\lambda$, RA and $\delta$ reported in the MWL, which represent those at maximum activity, that are within the initial and final values derived from SAAMER's observations. As it will be discussed in this section, due to either inconsistencies with the MWL or because the particular candidate was not present in the list, for a few particular streams we have also compared our results with the surveys reported by Younger et al. (2009) and, when possible due to their conjugate locations, Brown et al. (2010).

Table 1 presents the radiants for which identification was confirmed. The first two columns on this table provide the MWL shower name and IAU three letter code. The following 6 columns present the initial and final observed $\lambda$, RA and $\delta$. These are determined from the first and last days during which the relative strength of the shower is above the noise threshold (see Section 3 ). The last two columns in Table 1 present the measured temporal drifts obtained by fitting a line to the observed RA and $\delta$ as a function of $\lambda$ (see Fig. 5). Overall we have identified the presence of 32 shower radiants in the observations presented in this study, similar to the number of showers found in southern hemisphere survey reported by Younger et al. (2009). Brown et al. (2008), using CMOR also in single station mode identified 45 showers.

From the results shown in Table 1, it can be observed that for over $60 \%$ of the radiants found, the MWL values fall within the observed initial and final values for at least two of the three coordinates, giving us confidence that SAAMER's observations do indeed correspond to those showers. For three of the observed showers (APS, CAP and SDA), the MWL values are very close to those observed ones but some differences are evident. For the case of APS, the radiant was observed during $6^{\circ}$ in $\lambda$, a much shorter interval than the one observed by CMOR (Brown et al., 2010), and the final observed Solar Longitude is $13^{\circ}$ lower than the MWL value. Similarly for the case of SDA for which the expected maximum activity Solar Longitude value is $5^{\circ}$ lower than the initial value observed by SAAMER. The Aquariid complex, however, does get quite crowded with a number of different constituent streams around the same time that SDA peaks. It may be the active radiants classified as SDA includes other components.

For the case of MIC and ECR, although the observed Solar Longitudes as well as RA agree with the MWL values, the observed declinations are $5-7^{\circ}$ higher than the expected values. In particular, the ECR observed declination agrees with the values observed by Younger et al. (2009) utilizing meteor radars in Australia and Antarctica. This shower is located at a very high southern latitude and thus recent data are scarce. We hope that our future orbital determination will shed lights as to the origin of these differences. Similarly with the STA, although differences between the observed and 
Table 1

List of meteor shower radiants observed with SAAMER.

\begin{tabular}{|c|c|c|c|c|c|c|c|c|c|}
\hline Name & IAU Code & $\lambda_{i}$ & $\lambda_{f}$ & $R A_{i}$ & $R A_{f}$ & $\delta_{i}$ & $\delta_{f}$ & $\Delta R A$ & $\Delta \delta$ \\
\hline Daytime April Piscids & APS & 21 & 27 & 359 & 3.1 & 0.9 & 4.4 & 0.7 & 0.4 \\
\hline Daytime $\xi$ Cetids & XIC & 32 & 60 & 16.9 & 41.2 & -0.2 & 8.9 & 0.9 & 0.3 \\
\hline$\eta$ Aquarids & ETA & 35 & 59 & 329.6 & 346.5 & -4.5 & 3.6 & 0.7 & 0.3 \\
\hline Southern Daytime $\omega$ Cetids & OCE & 40 & 57 & 16.5 & 32.1 & -9.1 & -1.9 & 0.9 & 0.4 \\
\hline$\alpha$ Scorpiids & ASC & 58 & 61 & 249.7 & 250.2 & -28.4 & -29.8 & -0.1 & -0.2 \\
\hline Southern $\mu$ Sagitariids & SSG & 67 & 94 & 255.2 & 278.5 & -30.2 & -33.9 & 0.9 & -0.1 \\
\hline Daytime Arietids & ARI & 71 & 85 & 39.52 & 48.36 & 21.6 & 25.8 & 0.63 & 0.3 \\
\hline Southern June Aquiliids & $\mathrm{SZC}$ & 74 & 113 & 304.4 & 327.7 & -37.4 & -28 & 0.6 & 0.3 \\
\hline Northern June Aquiliids & NZC & 85 & 95 & 297.9 & 303.3 & -9.2 & -6.6 & 0.6 & 0.3 \\
\hline Southern $\sigma$ Sagitariids & SSS & 84 & 100 & 286.6 & 298 & -28.4 & -23.5 & 0.6 & 0.3 \\
\hline$\alpha$ Capricornids & CAP & 96 & 113 & 305.3 & 318.8 & -7.1 & -2.5 & 0.9 & 0.2 \\
\hline July Phoenicids & PHE & 100 & 123 & 20.6 & 43 & -55.2 & -40.1 & 0.8 & 0.7 \\
\hline Microscopiids & MIC & 108 & 125 & 311.8 & 327 & -23.1 & -21 & 0.9 & 0.08 \\
\hline$\sigma$ Capricornids & SCA & 110 & 128 & 297.3 & 304.9 & -15.1 & -11.2 & 0.5 & 0.2 \\
\hline Piscis Austrinids & PAU & 114 & 127 & 332.8 & 350.7 & -21.4 & -24 & 1.5 & -0.4 \\
\hline 99 Aquariids & NNA & 124 & 134 & 353.8 & 357.2 & -26.9 & -21.4 & 0.5 & 0.5 \\
\hline August $\beta$ Piscids & BPI & 126 & 167 & 325 & 359.9 & -10.8 & 1.9 & 0.9 & 0.3 \\
\hline Southern $\delta$ Aquarids & SDA & 130 & 141 & 342.8 & 352.7 & -17.4 & -14.3 & 0.8 & 0.3 \\
\hline Northern $\delta$ Aquarids & NDA & 126 & 138 & 342.1 & 345.7 & -3.5 & 0.9 & 0.2 & 0.3 \\
\hline$\omega$ Piscids & OPC & 162 & 172 & 0.4 & 5.9 & 1.5 & 3.6 & 0.5 & 0.2 \\
\hline Southern Taurids & STA & 178 & 212 & 17.6 & 43 & -0.06 & 7.2 & 0.8 & 0.2 \\
\hline Daytime Sextantids & DSX & 179 & 194 & 148.8 & 159.4 & 0.07 & -6.2 & 0.7 & -0.5 \\
\hline Orionids & ORI & 205 & 212 & 92.2 & 97.7 & 15.1 & 16 & 0.8 & 0.03 \\
\hline November $\omega$ Orionids & NOO & 241 & 246 & 86.5 & 90.4 & 14.4 & 14.4 & 0.8 & 0.002 \\
\hline Geminids & GEM & 259 & 262 & 110.1 & 113.6 & 30.3 & 30.5 & 1.1 & 0.03 \\
\hline$\eta$ Carinids & ECR & 280 & 291 & 159.4 & 169.3 & -51.5 & -53.3 & 0.9 & -0.2 \\
\hline$\zeta$ Puppids & ZPU & 234 & 240 & 124.6 & 127 & -45.4 & -43.9 & 0.3 & 0.09 \\
\hline$\gamma$ Puppids & PUP & 247 & 264 & 131.7 & 142.1 & -48.1 & -55.4 & 0.8 & -0.5 \\
\hline b Puppids & PVE & 274 & 276 & 139 & 140.6 & -49.5 & -51.2 & & \\
\hline January $\alpha$ Pixids & APY & 299 & 301 & 129.9 & 133.2 & -33.7 & -37.1 & 1.6 & -1.7 \\
\hline Daytime $\xi$ Sagitariids & XSA & 288 & 293 & 281.3 & 285.7 & -19.5 & -19.5 & 0.7 & 0.04 \\
\hline Daytime Chi Capricornids & DXC & 291 & 300 & 299.8 & 302.8 & -33.9 & -32 & 0.4 & 0.2 \\
\hline
\end{tabular}

expected declination exist, the observed ones agree well with those reported by Brown et al. (2010). The disagreement between multiple independent observations and the catalogue values may reflect limitations of the IAU MWL.

There were three observed well defined enhancements that occur within the temporal and spatial range of the Puppids complex. Without orbital or velocity information the definite correlation of which enhancement corresponds to which element of the complex cannot be done. We nevertheless report them separately to emphasize the fact that were spatially and temporally resolved. In addition, two radiants that agree with those reported by Younger et al. (2009) as Alpha Piscis Australids and Southern Piscids but are not listed in the MWL as such. The first radiant corresponds to the, previously unlisted, 99 Aquariids (NNA) shower (J. Jopek, Personal Communication, 2012) while the second one corresponds to the listed $\omega$ Piscids (OPC) shower (see Table 7, p. 726 in Jenniskens (2006), and shower 217 in IAU MWL). The names presented here follow the official nomenclature and should be refer as such in future reports (P. Jenniskens and T. Jopek, Personal Communication, 2012). Finally, Younger et al. (2009) reported nine previously undocumented showers, none of which were evident on the SAAMER's observations performed for this study. We will perform further search for these and other showers in the orbital data enabled by SAAMER's upgrades in the near future.

\section{Conclusions}

We presented in this manuscript an initial survey of meteor shower radiants in the southern hemisphere by applying the statistical methodology developed by Jones and Jones (2006) to the data collected during the first 4 years of SAAMER's operation as a single station radar. As described in Section 2, SAAMER is a new generation SKiYMET system designed with significant differ- ences from typical meteor radars including high transmitted power and an 8-antenna transmitting array enabling large detected rates at low zenith angles. We applied the statistical methodology developed by Jones and Jones (2006) to the data collected each day and compiled the results into 1 composite representative year at $1^{\circ}$ resolution in Solar Longitude. We then searched for enhancements in the activity which lasted for at least three consecutive Solar Longitudes and showed consistent positive drifts in RA and consistent drifts throughout their activity period in $\delta$, which were then single out for identification. Using this methodology, we have identified in our data 32 shower radiants, two of which were not part of the IAU commission 22 meteor shower working list. Preliminary analysis of SAAMER data had suggested that, due to the rapid changes in SAAMER's transmitted radiation pattern, which has nulls every $45^{\circ}$, with respect to all-sky meteor radar systems, meteor showers radiants would not be located properly using the Jones and Jones (2006) methodology. The resulting radiant could be shifted up to $20^{\circ}$ from their known location (M. Campbell-Brown and W. Cook, Personal Communication, 2010). In addition, because it transmits higher power in a relatively smaller volume, SAAMER has the potential to be more sensitive to lower mass meteoroids and in principle, less effective at detecting meteor showers, similar AMOR (Galligan and Baggaley, 2002b; Brown et al., 2008). The results presented here show, not only that this is not the case, but also that SAAMER is at least as effective as previous studies using traditional lower-power all-sky systems (Brown et al., 2008; Younger et al., 2009). It is also important to note from Table 1 the range of ecliptic latitudes that SAAMER enables to survey. It can effectively observe radiants from the ecliptic south pole to $\sim 30^{\circ} \mathrm{N}$ (e.g. Geminids), and thus once the orbital elements are accessible in future surveys will enable the detailed study of showers at high southern latitudes (e.g July Phoenicids or Puppids complex), which are unobservable from the CMOR's location. 


\section{Acknowledgments}

This work was supported by NSF Awards AGS - 0634650, AGS 0944104 and AST - 0908118. We wish to thank the EARG personnel for their invaluable help with the operation of SAAMER and D. Moser, P. Brown and M. Campbell-Brown for useful discussions.

\section{References}

Baggaley, W.J., Bennett, R.G.T., Steel, D.I., Taylor, A.D., 1994. The advance meteor orbit radar facility: Amor. Q. J. R. Astron. Soc. (35), 293-320.

Borovička, J., Koten, P., Spurny,, P., Bočcek, J., Štork, R., 2005. A survey of meteor spectra and orbits: evidence for three populations of Na-free meteoroids. Icarus 174 (March), 15-30. http://dx.doi.org/10.1016/j.icarus.2004.09.011.

Brown, P., Weryk, R.J., Wong, D.K., Jones, J., 2008. A meteoroid stream survey using the Canadian Meteor Orbit Radar. I: Methodology and radiant catalogue. Icarus 195, 317-339. http://dx.doi.org/10.1016/j.icarus.2007.12.002.

Brown, P., Wong, D.K., Weryk, R.J., Wiegert, P., 2010. A meteoroid stream survey using the Canadian Meteor Orbit Radar. II: Identification of minor showers using a 3D wavelet transform. Icarus 207, 66-81. http://dx.doi.org/10.1016 j.icarus.2009.11.015

Fritts, D.C., Janches, D., Hocking, W.K., 2010a. Southern Argentina Agile Meteor Radar: Initial assessment of Gravity Wave momentum fluxes. J. Geophys. Res. Atmospheres 115, 19123-+. http://dx.doi.org/10.1029/2010JD013891.

Fritts, D.C., Janches, D., Hocking, W.K., Mitchell, N.J., Taylor, M.J., 2012. Assessment of Gravity Wave momentum flux measurement capabilities by meteor radars having different transmitter power and antenna configurations. J. Geophys. Res. - Atmospheres 117, D10108. http://dx.doi.org/10.1029/2011JD017174.

Fritts, D. C., et al. (2010b), Southern Argentina Agile Meteor Radar: System design and initial measurements of large-scale winds and tides, J. Geophys. Res., 115, D18112, http://dx.doi.org/10.1029/2010JD013850.

Galligan, D.P., Baggaley, W.J., 2002. Wavelet enhancement for detecting shower structure in radar meteoroid data I. Methodology. In: IAU Colloq. 181: Dust in the Solar System and Other Planetary Systems.

Galligan, D.P., Baggaley, W.J., 2002. Wavelet enhancement for detecting shower structure in radar meteoroid data II. Application to the AMOR data. In: IAU Colloq. 181: Dust in the Solar System and Other Planetary Systems.

Hocking, W.K., Thayaparan, T., Jones, J., 1997. Meteor decay times and their use in determining a diagnostic mesospheric temperature-pressure parameter: Methodology and one year of data. Geophys. Res. Lett. 24, 2977-2980. http:// dx.doi.org/10.1029/97GL03048.
Hocking, W.K., Fueller, B., Vandepeer, B., 2001. Real-time determination of meteorrelated parameters utilizing modern digital technology. J. Atmos. Solar Terr. Phys 63, 155-169.

Jenniskens, P., 2006. Meteor Showers and their Parent Comets. Cambridge University Press.

Jenniskens, P., 2008. The Parent Bodies of our Meteor Showers. LPI Contributions.

Johansen, A., Oishi, J.S., Mac Low, M.-M., Klahr, H., Henning, T., Youdin, A., 2007. Rapid planetesimal formation in turbulent circumstellar disks. Nature 448, 1022-1025. http://dx.doi.org/10.1038/nature06086.

Jones, J., Jones, W., 2006. Meteor radiant activity mapping using single-station radar observations. Month. Not. R. Astron. Soc. 367, 1050-1056. http://dx.doi.org/ $10.1111 / \mathrm{j} .1365-2966.2006 .10025 . x$.

Jones, J., Morton, J.D., 1977. The determination of meteor stream radiants from single station observations. Bull. Astron. Inst. Czechoslovakia 28, 267-272.

Jones, J., Webster, A.R., Hocking, W.K., 1998. An improved interferometer design for use with meteor radars. Radio Sci. 33, 55-65. http://dx.doi.org/10.1029/ 97RS03050.

Jopek, T.J., Jenniskens, P.M., 2011. The working group on meteor showers nomenclature: A history, current status and a call for contributions. In: Cooke, W.J., Moser, D.E., Hardin, B.F., Janches, D. (Eds.), Meteoroids: The Smallest Solar System Bodies, NASA/CP-2011-216469, p. 7.

Malhotra, R., 1995. The origin of Pluto's orbit: Implications for the Solar System Beyond Neptune. Astron. J. 110, 420-429. http://dx.doi.org/10.1086/117532.

Nesvorný, D., Jenniskens, P., Levison, H.F., Bottke, W.F., Vokrouhlický, D., Gounelle, M., 2010. Cometary origin of the zodiacal cloud and carbonaceous micrometeorites. Implications for hot debris disks. Apstrophys. J. 713, 816836. http://dx.doi.org/10.1088/0004-637X/713/2/816.

Sykes, M.V., Walker, R.G., 1992. Cometary dust trails. I - Survey. Icarus 95, 180-210. http://dx.doi.org/10.1016/0019-1035(92)90037-8.

Walsh, K.J., Morbidelli, A., Raymond, S.N., O’Brien, D.P., Mandell, A.M., 2011. A low mass for Mars from Jupiter's early gas-driven migration. Nature 475, 206-209. http://dx.doi.org/10.1038/nature10201.

Wiegert, P., Brown, P., 2004. The problem of linking minor meteor showers to their parent bodies: Initial considerations. Earth Moon Planets 95, 19-26. http:// dx.doi.org/10.1007/s11038-005-4342-8.

Wiegert, P., Vaubaillon, J., Campbell-Brown, M., 2009. A dynamical model of the sporadic meteoroid complex. Icarus 201, 295-310. http://dx.doi.org/10.1016/ j.icarus.2008.12.030

Younger, J.P., Reid, I.M., Vincent, R.A., Holdsworth, D.A., Murphy, D.J., 2009. A southern hemisphere survey of meteor shower radiants and associated stream orbits using single station radar observations. Month. Not. R. Astron. Soc. 398 350-356. http://dx.doi.org/10.1111/j.1365-2966.2009.15142.x. 